\title{
Lakshmi - Manoj generalized Yang-Fourier transforms to heat-conduction in a semi-infinite fractal bar
}

\author{
Lakshmi Narayan Mishra ${ }^{1,2}$, Manoj Sharma ${ }^{3}$, Vishnu Narayan Mishra ${ }^{4}$ \\ ${ }^{1}$ Department of Mathematics, National Institute of Technology, Silchar - 788 010, District - Cachar (Assam), India \\ ${ }^{2}$ L. 1627 Awadh Puri Colony Beniganj, Phase - III, Opposite - Industrial Training Institute (I. T. I.), Faizabad - 224001 (Uttar Pradesh), \\ India \\ ${ }^{3}$ Department of Mathematics, RJIT, BSF Academy, Tekanpur, Gwalior (M.P.), India \\ ${ }^{4}$ Applied Mathematics and Humanities Department, Sardar Vallabhbhai National Institute of Technology, Ichchhanath Mahadev Dumas Road, \\ Surat - 395007 (Gujarat), India
}

\section{Email address:}

lakshminarayanmishra04@gmail.com (L. N. Mishra),manoj240674@yahoo.co.in (M. Sharma), vishnunarayanmishra@gmail.com (V. N. Mishra)

\section{To cite this article:}

Lakshmi Narayan Mishra, Manoj Sharma, Vishnu Narayan Mishra. Lakshmi - Manoj Generalized Yang-Fourier Transforms to HeatConduction in a Semi-Infinite Fractal Bar. Pure and Applied Mathematics Journal. Vol. 4, No. 2, 2015, pp. 57-61. doi: 10.11648/j.pamj.20150402.15

\begin{abstract}
In the present era, fractional calculus plays an important role in various fields. Fractional Calculus is a field of mathematic study that grows out of the traditional definitions of the calculus integral and derivative operators in much the same way fractional exponents is an outgrowth of exponents with integer value. Based on the wide applications in engineering and sciences such as physics, mechanics, chemistry, and biology, research on fractional ordinary or partial differential equations and other relative topics is active and extensive around the world. In the past few years, the increase of the subject is witnessed by hundreds of research papers, several monographs, and many international conferences. The purpose of present paper to solve 1-D fractal heat-conduction problem in a fractal semi-infinite bar has been developed by local fractional calculus employing the analytical Manoj Generalized Yang-Fourier transforms method.
\end{abstract}

Keywords: Fractal Bar, Heat-Conduction Equation, Lakshmi-Manoj Generalized Yang-Fourier Transforms, Yang-Fourier Transforms, Local Fractional Calculus

\section{Introduction}

Manoj Generalized Yang-Fourier transforms which is obtained by authors by generalization of Yang-Fourier transforms is a technique of fractional calculus for solving mathematical, physical and engineering problems. The fractional calculus is continuously growing in last five decades [1-7]. Most of the fractional ordinary differential equations have exact analytic solutions, while others required either analytical approximations or numerical techniques to be applied, among them: fractional Fourier and Laplace transforms [8, 41], heat-balance integral method [9-11], variation iteration method (VIM) [12-14], decomposition method $[15,41]$, homotopy perturbation method $[16,41]$ etc.

The problems in fractal media can be successfully solved by local fractional calculus theory with problems for nondifferential functions [25-32]. Local fractional differential equations have been applied to model complex systems of fractal physical phenomena [30-41] local fractional Fourier series method [38], Yang-Fourier transform [39, 40, 41]

\section{Generalized Yang-Fourier Transform and Its Properties}

Let us Consider $f(x)$ is local fractional continuous in $(-\infty, \infty)$ we denote as $f(x) \in C a, \beta(-\infty, \infty) \quad[32,33,35]$.

Let $f(x) \in C a, \beta(-\infty, \infty)$ The Generalized Yang-Fourier transform developed by authors is written in the form [30, 31 , 39, 40, 41]:

$F_{\alpha, \beta}\{f(x)\}=f_{\omega}^{F, \alpha, \beta}(\omega)=$ 
$\frac{(\delta)_{\beta}}{\Gamma(1+\alpha+\beta)} \int_{-\infty}^{\infty} E_{\alpha, \beta}^{\delta}\left(-i^{\alpha+\beta} \omega^{\alpha+\beta} x^{\alpha+\beta}\right) f(x)(d x)^{\alpha+\beta}$.

When we put $\beta$ equal to zero, it converts in to the YangFourier transform [41].

Then, the local fractional integration is given by [30-32, $35-37,41]$ :

$$
\begin{aligned}
& \frac{(\delta)_{\beta} 1}{\Gamma(1+\alpha+\beta)} \int_{a}^{b} f(t)(d x)^{\alpha+\beta}= \\
& \frac{(\delta)_{\beta}}{\Gamma(1+\alpha+\beta)} \lim _{\Delta t \rightarrow 0} \sum_{j=0}^{j=N-1} f\left(t_{j}\right)\left(\Delta t_{j}\right)^{\alpha+\beta}
\end{aligned}
$$

where $\Delta t_{j}=t_{j+1}-t_{j}, \Delta t=\max \left\{\Delta t_{1}, \Delta t_{2}, \Delta t_{j}, \ldots\right\}$ and $\left\{t_{j}, t_{j+1}\right\}, j=0, \ldots, N-1, t_{0}=\mathrm{a}, t_{N}=\mathrm{b}$, is a partition of the interval $[a, b]$.

If $F_{\alpha, \beta}\{f(x)\}=f_{\omega}^{F, \alpha, \beta}(\omega)$, then its inversion formula takes the form $[30,31,39,40,41]$

$f(x)=F_{\alpha, \beta}^{-1}\left[f_{\omega}^{F, \alpha, \beta}(\omega)\right]=$

$\frac{(\delta)_{\beta}}{(2 \pi)^{\alpha+\beta}} \int_{-\infty}^{\infty} E^{\delta}{ }_{\alpha, \beta}\left(-i^{\alpha+\beta} \omega^{\alpha+\beta} x^{\alpha+\beta}\right) f_{\omega}^{F, \alpha, \beta}(\omega)(d \omega)^{\alpha+\beta}$,

when we put $\beta$ equal to zero, it converts in to the Yang Inverse Fourier transform [41].

Some properties are shown as it follows [30, 31]:

Let $\quad F_{\alpha, \beta}\{f(x)\}=f_{\omega}^{F, \alpha, \beta}(\omega), \quad$ and $\quad F_{\alpha, \beta}\{g(x)\}=$ $f_{\omega}^{F, \alpha, \beta}(\omega)$, and let be two constants, if $(\delta)_{0}$. Then we have:

$$
F_{\alpha, \beta}\{c f(x)+d g(x)\}=c F_{\alpha, \beta}\{f(x)\}+d F_{\alpha, \beta}\{g(x)\}
$$

If $\lim _{|x| \rightarrow \infty} f(x)=0$, then we have:

$$
F_{\alpha, \beta}\left\{f^{\alpha, \beta}(x)\right\}=i^{\alpha+\beta} \omega^{\alpha+\beta} F_{\alpha, \beta}\{f(x)\}
$$

In equation (5) the local fractional derivative is defined as:

$$
f^{\alpha, \beta}\left(x_{0}\right)=\left.\frac{d^{\alpha+\beta} f(x)}{d x^{\alpha+\beta}}\right|_{x=x_{0}}=\lim _{x \rightarrow x_{0}} \frac{\Delta^{\alpha+\beta}\left[f(x)-f\left(x_{0}\right)\right]}{\left(x-x_{0}\right)^{\alpha+\beta}}
$$

where

$$
\Delta^{\alpha+\beta}\left[f(x)-f\left(x_{0}\right)\right] \cong \Gamma(1+\alpha+\beta) \Delta\left[f(x)-f\left(x_{0}\right)\right],
$$

As a direct result, repeating this process, when:

$$
\begin{gathered}
f(0)=f^{\alpha, \beta}(0)=\cdots=f^{(k-1) \alpha,(k-1) \beta}(0)=0 \\
F_{\alpha, \beta}\left\{f^{k \alpha, k \beta}(x)\right\}=i^{\alpha+\beta} \omega^{\alpha+\beta} F_{\alpha, \beta}\{f(x)\}
\end{gathered}
$$

\section{Heat Conduction in a Fractal Semi-Infinite Bar}

If a fractal body is subjected to a boundary perturbation, then the heat diffuses in depth modeled by a constitutive relation where the rate of fractal heat flux $\bar{q}(x, y, z, t)$ is proportional to the local fractional gradient of the temperature [32,41], namely:

$$
\bar{q}(x, y, z, t)=-K^{2 \alpha+2 \beta} \nabla^{\alpha+\beta} T(x, y, z, t) .
$$

Here the pre-factor $K^{2 a+2 \beta}$ is the thermal conductivity of the fractal material. Therefore, the fractal heat conduction equation without heat generation was suggested in [32] as:

$K^{2 \alpha+2 \beta} \frac{d^{2(\alpha+\beta)} T(x, y, z, t)}{d x^{2(\alpha+\beta)}}-\rho_{\alpha+\beta} c_{\alpha+\beta} \frac{d^{2(\alpha+\beta)} T(x, y, z, t)}{d x^{2(\alpha+\beta)}}=0$,

where $\rho_{\alpha+\beta}$ and $c_{\alpha+\beta}$ are the density and the specific heat of material, respectively.

The fractal heat-conduction equation with a volumetric heat generation $g(x, y, z, t)$ can be described as $[32,41]$ :

$K^{2 \alpha+2 \beta} \nabla^{2 \alpha+2 \beta} T(x, y, z, t)+$

$g(x, y, z, t) \rho_{\alpha+\beta} c_{\alpha+\beta} \frac{\partial^{(\alpha+\beta)} T(x, y, z, t)}{\partial t^{(\alpha+\beta)}}$

The 1-D fractal heat-conduction equation $[32,41]$ reads as: $K^{2 \alpha+2 \beta} \frac{\partial^{2(\alpha+\beta)} T(x, t)}{\partial x^{2(\alpha+\beta)}}-\rho_{\alpha+\beta} c_{\alpha+\beta} \frac{\partial^{(\alpha+\beta)} T(x, t)}{\partial t^{(\alpha+\beta)}}=0,0<x<\infty$, $t>0$

with initial and boundary conditions are:

$$
\frac{\partial^{(\alpha+\beta)} T(0, t)}{\partial t^{(\alpha+\beta)}}=E_{\alpha+\beta}^{\delta} t^{\alpha+\beta}, T(0, t)=0
$$

The dimensionless forms of $(12 \mathrm{a}, \mathrm{b})$ are $[35,41]$ :

$$
\begin{gathered}
\frac{\partial^{2(\alpha+\beta)} T(x, t)}{\partial x^{2(\alpha+\beta)}}=\frac{\partial^{(\alpha+\beta)} T(x, t)}{\partial x^{(\alpha+\beta)}}=0 \\
\frac{\partial^{(\alpha+\beta)} T(0, t)}{\partial x^{(\alpha+\beta)}}=E_{\alpha+\beta}^{\delta} t^{\alpha+\beta}, T(0, t)=0 .
\end{gathered}
$$

Based on equation (12a), the local fractional model for 1-D fractal heat-conduction in a fractal semi-infinite bar with a source term $g(x, t)$ is:

$$
\begin{gathered}
K^{2 \alpha+2 \beta} \frac{\partial^{2(\alpha+\beta)} T(x, t)}{\partial x^{2(\alpha+\beta)}}-\rho_{\alpha+\beta} c_{\alpha+\beta} \frac{\partial^{(\alpha+\beta)} T(x, t)}{\partial t^{(\alpha+\beta)}}=g(x, t), \\
-\infty<x<\infty, t>0
\end{gathered}
$$

with

$$
T(x, 0)=f(x),-\infty<x<\infty .
$$

The dimensionless form of the model $(14 a, b)$ is:

$$
\begin{gathered}
\frac{\partial^{2(\alpha+\beta)} T(x, t)}{\partial x^{2(\alpha+\beta)}}=\frac{\partial^{(\alpha+\beta)} T(x, t)}{\partial t^{(\alpha+\beta)}}=0,-\infty<x<\infty, t>0 \\
T(x, 0)=f(x),-\infty<x<\infty .
\end{gathered}
$$

\section{Solutions by the Generalized Yang-Fourier Transform Method}

Let us consider that $F_{\alpha, \beta}\{T(x, t)\}=T_{\omega}^{F, \alpha, \beta}(\omega, t)$ is the Generalized Yang-Fourier transform of $T(x, t)$, regarded as a non-differentiable function of $x$. Applying the Yang-Fourier transform to the first term of equation (15a), we obtain:

$$
\begin{gathered}
F_{\alpha, \beta}\left\{\frac{\partial^{2(\alpha+\beta)} T(x, t)}{\partial x^{2(\alpha+\beta)}}\right\}=\left(i^{2(\alpha+\beta)} \omega^{2(\alpha+\beta)}\right) T_{\omega}^{F, \alpha, \beta}(\omega, t)= \\
\omega^{2(\alpha+\beta)} T_{\omega}^{F, \alpha, \beta}(\omega, t) .
\end{gathered}
$$

On the other hand, by changing the order of the local 
fractional differentiation and integration in the second term of equation (15a), we get:

$$
F_{\alpha, \beta}\left\{\frac{\partial^{2(\alpha+\beta)}}{\partial t^{2(\alpha+\beta)}} T(x, t)\right\}=\frac{\partial^{(\alpha+\beta)}}{\partial t^{(\alpha+\beta)}} T_{\omega}^{F, \alpha, \beta}(\omega, t) .
$$

For the initial value condition, the Yang-Fourier transform provides:

$$
F_{\alpha, \beta}\{T(x, 0)\}=T_{\omega}^{F, \alpha, \beta}(\omega, 0)=F_{\alpha, \beta}\{f(x)\}=f_{\omega}^{F, \alpha, \beta}(\omega) .
$$

Thus we get from equation. (16a, b, c):

$$
\begin{aligned}
& \qquad T(x, t)=\frac{(\delta)_{\beta}}{(2 \pi)^{\alpha+\beta}} \int_{-\infty}^{\infty} E^{\delta} \\
& M_{\omega}^{F, \alpha, \beta}(\omega)=\frac{1}{(2 \pi)^{\alpha+\beta}} E_{\alpha, \beta}^{\delta}\left(-\omega^{2}\right. \\
& \text { From [30, 32] we obtain, } \\
& F_{\alpha+\beta}\left\{E_{\alpha+\beta}^{\delta}\left(-\frac{\omega^{2(\alpha+\beta)}}{C^{2(\alpha+\beta)}}\right)\right\}= \\
& \frac{C^{(\alpha+\beta)} \frac{\pi^{\frac{\alpha+\beta}{2}}}{\Gamma(1+\alpha+\beta)}}{\Gamma} E^{\delta} E_{\alpha, \beta}\left(-\frac{C^{2(\alpha+\beta)} \omega^{2(\alpha+\beta)}}{4^{(\alpha+\beta)}}\right) .
\end{aligned}
$$$$
M_{\omega}^{F, \alpha, \beta}(\omega)=\frac{1}{(2 \pi)^{\alpha+\beta}} E_{\alpha, \beta}^{\delta}\left(-\omega^{2(\alpha+\beta)} t^{\alpha+\beta}\right) .
$$

Let $C^{2(\alpha+\beta)} / 4^{\alpha+\beta}=t^{\alpha+\beta}$. Then we get:

$F_{\alpha+\beta}\left\{E_{\alpha+\beta}^{\delta}\left(-\frac{\omega^{2(\alpha+\beta)}}{4^{\alpha+\beta} t^{\alpha+\beta}}\right)\right\}=$

$\frac{4^{\alpha+\beta} t^{\frac{\alpha+\beta}{2}} \pi^{\frac{\alpha+\beta}{2}}}{\Gamma(1+\alpha+\beta)} E_{\alpha, \beta}^{\delta}\left(-\omega^{2(\alpha+\beta)} t^{\alpha+\beta}\right)=$

$(\delta)_{\beta} \frac{4^{\alpha+\beta} t^{\frac{\alpha+\beta}{2}} \pi^{\frac{\alpha+\beta}{2}}}{\Gamma(1+\alpha+\beta)}(2 \pi)^{\alpha+\beta} M_{\omega}^{F, \alpha, \beta}(\omega)$

Thus, $M_{\omega}^{F, \alpha, \beta}(\omega)$ have the inverse

$\frac{(\delta)_{\beta}}{(2 \pi)^{\alpha+\beta}} \int_{-\infty}^{\infty} E_{\alpha, \beta}^{\delta}\left(i^{\alpha+\beta} \omega^{\alpha+\beta} x^{\alpha+\beta}\right) M_{\omega}^{F, \alpha, \beta}(\omega)(d \omega)^{\alpha+\beta}=$
$=\frac{\Gamma(1+\alpha+\beta)}{4^{\alpha+\beta} t^{\frac{\alpha+\beta}{2}} \pi^{\frac{\alpha+\beta}{2}}} \frac{(\delta)_{\beta^{1}}}{(2 \pi)^{\alpha+\beta}} E_{\alpha+\beta}^{\delta}\left(-\frac{\omega^{2(\alpha+\beta)}}{4^{\alpha+\beta} t^{\alpha+\beta}}\right)$.

Hence, we get:

$T(x, t)=(M f)(x)=$

$(\delta)_{\beta} \frac{\Gamma(1+\alpha+\beta)}{4^{\alpha+\beta} t^{\frac{\alpha+\beta}{2}} \pi^{\frac{\alpha+\beta}{2}}} \int_{-\infty}^{\infty} f(\xi) E_{\alpha, \beta}^{\delta}\left(-\frac{(x-\xi)^{2(\alpha+\beta)}}{4^{\alpha+\beta} t^{\alpha+\beta}}\right)(d \xi)^{\alpha+\beta}$.

Special case:

If we take $\beta=0$ then the results of generalized Yang Fourier Transforms convert in Yang Fourier Transforms results [41].

\section{Conclusions}

The communication, presented an analytical solution of 1$\mathrm{D}$ heat conduction in fractal semi-infinite bar by the Generalized Yang-Fourier transform of non-differentiable functions. Some important interesting applications can be seen in $([42]-[46])$.

\section{Acknowledgments}

The authors would like to express their deep gratitude to $\frac{\partial^{(\alpha+\beta)}}{\partial t^{(\alpha+\beta)}} T_{\omega}^{F, \alpha, \beta}(\omega, t)+\omega^{2(\alpha+\beta)} T_{\omega}^{F, \alpha, \beta}(\omega, t)=$ $0, T_{\omega}^{F, \alpha, \beta}(\omega, 0)=f_{\omega}^{F, \alpha, \beta}(\omega)$.

This is an initial value problem of a local fractional differential equation with $t$ as independent variable and $w$ as a parameter.

$$
T(\omega, t)=f_{\omega}^{F, \alpha, \beta}(\omega) E_{\alpha, \beta}^{\delta}\left(-\omega^{2(\alpha+\beta)} t^{\alpha+\beta}\right) .
$$

Consequently, using inversion formula, equation (3), we obtain:

$(\omega) E_{\alpha, \beta}^{\delta}\left(-\omega^{2(\alpha+\beta)} t^{\alpha+\beta}\right)(d \omega)^{\alpha+\beta}$

the anonymous learned referee(s) and the editor for their valuable suggestions and constructive comments, which resulted in the subsequent improvement of this research paper. The authors are also grateful to all the editorial board members and reviewers of Pure and Applied Mathematics journal. The first author Lakshmi Narayan Mishra acknowledges the Ministry of Human Resource Development (MHRD), New Delhi, India, for supporting this research article at the Department of Mathematics, National Institute of Technology (NIT), Silchar, Assam. The third author Vishnu Narayan Mishra acknowledges that this paper was supported by Sardar Vallabhbhai National Institute of Technology (SVNIT), Surat (Gujarat), India. Each author contributed equally in the development of the paper. The second author Manoj Sharma conceived of the study and participated in its design and coordination. All the authors drafted the manuscript, participated in the sequence alignment, read and approved the final version of manuscript.

\section{References}

[1] Kilbas, A.A., Srivastava H M., Trujillo, J J.; Theory and Applications of Fractional Differential Equations, Elsevier Science, Amsterdam, 2006.

[2] Mainardi, F., Fractional Calculus and Waves in Linear Viscoelasticity, Imperial College Press, London, 2010.

[3] Podlubny, I., Fractional Differential Equations, Academic Press, New York, USA, 1999.

[4] Klafter, J., et al., (Eds.), Fractional Dynamics in Physics: Recent Advances, World Scientific, Singapore, 2012.

[5] Zaslavsky, G.M., Hamiltonian Chaos and Fractional Dynamics, Oxford University Press, Oxford, UK, 2005.

[6] West, B, M. Bologna, and P. Grigolini, Physics of Fractal Operators, Springer, New York, USA, 2003.

[7] Carpinteri, A., Mainardi, F., (Eds.), Fractals and Fractional Calculus in Continuum Mechanics, Springer, Wiena, 1997.

[8] Baleanu, D., Golmankhaneh AK,; Golmankhaneh AK, Fractional Calculus Models and Numerical Methods, Complexity, Nonlinearity and Chaos, World Scientific, Singapore, 2012.

[9] Hristov, J., Heat-Balance Integral to Fractional (Half-Time) Heat Diffusion Sub-Model, Thermal Science, 14 (2010), 2, pp. 291-316. 
[10] Hristov, J., Integral-Balance Solution to the Stokes' First Problem of a Viscoelastic Generalized Second Grade Fluid, Thermal Science, 16 (2012), 2, pp. 395-410.

[11] Hristov, J., Transient Flow of a Generalized Second Grade Fluid Due to a Constant Surface Shear Stress: An Approximate Integral-Balance Solution, Int. Rev. Chem. Eng., 3 (2011), 6, pp. 802-809.

[12] Jafari, H., Tajadodi, H; A Modified Variational Iteration Method for Solving Fractional Riccati Differential Equation by Adomian Polynomials, Fractional Calculus and Applied Analysis, 16 (2013), 1, pp. 109-122.

[13] Wu, G. C., Baleanu, D., Variational Iteration Method for Fractional Calculus-a Universal Approach by Laplace Transform, Advances in Difference Equations, 2013 (2013), 1, pp. 1-9.

[14] Ates, I., Yildirim, A., Application of Variational Iteration Method to Fractional Initial-Value Problems, Int. J. Nonl. Sci. Num. Sim., 10 (2009), 7, pp. 877-884.

[15] Duan, J. S., et al., Solutions of the Initial Value Problem for Nonlinear Fractional Ordinary Differential Equations by the Rach-Adomian-Meyers Modified Decomposition Method, Appl. Math. Comput., 218 (2012), 17, pp. 8370-8392.

[16] Momani, S., Yildirim, A., Analytical Approximate Solutions of the Fractional Convection-Diffusion Equation with Nonlinear Source Term by He's Homotopy Perturbation Method, Int. J. Comp. Math. Sci., 87 (2010), 5, pp. 10571065 .

[17] Guo, S., Mei, L, Li, Y., Fractional Variational Homotopy Perturbation Iteration Method and Its Application to a Fractional Diffusion Equation, Appl. Math. Comput., 219 (2013), 11, pp. 5909-5917.

[18] Sun, H. G., et al., A Semi-Discrete Finite Element Method for a Class of Time-Fractional Diffusion Equations, Phil. Trans. Royal Soc. A: 371 (2013), 1990, pp. 1471-2962.

[19] Jafari, H., et al., Fractional Subequation Method for CahnHilliard and Klein-Gordon Equations, Abstract and Applied Analysis, 2013 (2013), Article ID 587179.

[20] Luchko,Y., Kiryakova, V., The Mellin Integral Transform in Fractional Calculus, Fractional Calculus and Applied Analysis, 16 (2013), 2, pp .405-430.

[21] Abbasbandy, S., Hashemi, M. S., On Convergence of Homotopy Analysis Method and its Application to Fractional integro-Differential Equations, Quaestiones Mathematicae, 36 (2013), 1, pp. 93-105.

[22] Hashim, I., et al., Homotopy Analysis Method for Fractional IVPs, Comm. Nonl. Sci. Num. Sim., 14 (2009), 3, pp. 674-684.

[23] Li, C., Zeng, F., The Finite Difference Methods for Fractional Ordinary Differential Equations, Num. Func. Anal. Optim., 34 (2013), 2, pp. 149-179.

[24] Demir, A., et al., Analysis of Fractional Partial Differential Equations by Taylor Series Expansion, Boundary Value Problems, 2013 (2013), 1, pp. 68-80.

[25] Kolwankar, K. M., Gangal, A. D., Local Fractional FokkerPlanck Equation, Physical Review Letters, 80 (1998), 2, pp. 214-217.

[26] Chen, W., Time-Space Fabric Underlying Anomalous
Diffusion, Chaos, Solitons \& Fractals, 28 (2006), 4, pp. 923929.

[27] Fan, J., He, J.- H., Fractal Derivative Model for Air Permeability in Hierarchic Porous Media, Abstract and Applied Analysis, 2012 (2012), Article ID 354701.

[28] Jumarie, G., Probability Calculus of Fractional Order and Fractional Taylor's Series Application to Fokker-Planck Equation and Information of Non-Random Functions, Chaos, Solitons \& Fractals, 40 (2009), 3, pp. 1428-1448.

[29] Carpinteri, A., Sapora, A., Diffusion Problems in Fractal Media Defined on Cantor Sets, ZAMM, 90 (2010), 3, pp. 203 210.

[30] Yang, X. J., Local Fractional Functional Analysis and Its Applications, Asian Academic publisher Limited, Hong Kong, 2011.

[31] Yang, X. J., Local Fractional Integral Transforms, Progress in Nonlinear Science, 4 (2011).

[32] Yang, X. J., Advanced Local Fractional Calculus and Its Applications, World Science Publisher, New York, USA, 2012.

[33] Su,W. H., et al., Fractional Complex Transform Method for Wave Equations on Cantor Sets within Local Fractional Differential Operator, Advances in Difference Equations, 2013 (2013), 1, pp. 97-103.

[34] Yang Xiaojun, Hu, M. S., Baleanu, D., One-Phase Problems for Discontinuous Heat Transfer in Fractal Media, Mathematical Problems in Engineering, 2013 (2013), Article ID $358473,2013$.

[35] Yang, X, J., Baleanu, D., Fractal Heat Conduction Problem Solved by Local Fractional Variation Iteration Method, Thermal Science, 17 (2013), 2, pp. 625-628.

[36] Yang, Y., J., Dumitru Baleanu, Xiao-Jun Yang., A Local Fractional Variational Iteration Method for Laplace Equation within Local Fractional Operators, Abstract and Applied Analysis, 2013 (2013), Article ID 202650.

[37] $\mathrm{Su}, \mathrm{W} . \mathrm{H}$., et al., Damped Wave Equation and Dissipative Wave Equation in Fractal Strings within the Local Fractional Variational Iteration Method, Fixed Point Theory and Applications, 2013 (2013), 1, pp. 89-102.

[38] Hu, M. S., et al., Local Fractional Fourier Series with Application to wave Equation in Fractal Vibrating String, Abstract and Applied Analysis, 2012 (2012), Article ID 567401

[39] Zhong, W. P., et al., Applications of Yang-Fourier Transform to Local Fractional Equations with Local Fractional Derivative and Local Fractional Integral, Advanced Materials Research, 461 (2012), pp. 306-310.

[40] He, J.-H., Asymptotic Methods for Solitary Solutions and Compactions, Abstract and Applied Analysis, 2012 (2012), Article ID 916793.

[41] Yang, Ai-M, Zhang, Y-Z, Long, Y, the Yang-Fourier transforms to heat-conduction in a semi-infinite fractal bar, Thermal Science, 2013, vol. 17, no. 3, pp.707-713.

[42] Deepmala, A study on fixed point theorems for nonlinear contractions and its applications [Ph.D. thesis], Pt. Ravishankar Shukla University, Raipur, India, 2014. 
[43] Deepmala, "Existence theorems for solvability of a functional equation arising in dynamic programming," International Journal ofMathematics andMathematical Sciences, vol. 2014,Article ID706585, 9 pages, 2014.

[44] V. N. Mishra, K. Khatri, L. N. Mishra, and Deepmala, "Inverse result in simultaneous approximation by BaskakovDurrmeyer Stancu operators," Journal of Inequalities and Applications, vol. 2013, article 586, 2013.
[45] V.N. Mishra, Some Problems on Approximations of Functions in Banach Spaces, Ph.D. Thesis (2007), Indian Institute of Technology, Roorkee - 247 667, Uttarakhand, India.

[46] Deepmala and H. K. Pathak, A study on some problems on existence of solutions for nonlinear functional-integral equations, Acta Mathematica Scientia, 33 B(5) (2013), 13051313. 